\title{
INCLUSIÓN SOCIAL, CONSTRUCCIÓN DE CIUDADANÍA Y FORTALECIMIENTO COMUNITARIO, COMO PERSPECTIVA DE INTERVENCIÓN DE LA PSICOLOGÍA SOCIAL COMUNITARIA LATINOAMERICANA
}

\author{
Hugo Sanabria Tovar ${ }^{22}$
}

\begin{abstract}
RESUMEN
El artículo aborda inicialmente el situado histórico de la psicología social comunitaria en Latinoamérica y en Colombia como resultado del proceso dialéctico al cual está sometida toda realidad bien sea esta referida a los procesos que se dan en la naturaleza o ya los construidos por personas y comunidades como son los sociales. Igualmente en este sucinto recorrido histórico se hace referencia a la perspectiva ético - política que ha venido asumiendo la psicología social comunitaria a favor de los sectores vulnerables y socialmente excluidos. Asunto que la ha llevado a que dentro de su agenda se prioricen algunos procesos psicosociales como son la Inclusión social, construcción de ciudadanía y fortalecimiento comunitario, como potenciadores del agenciamiento en la transformación personal y comunitaria a través de sus propios actores sociales.
\end{abstract}

Palabras clave: psicología social comunitaria, inclusión social, construcción de ciudadanía, fortalecimiento comunitario.

\begin{abstract}
This article alludes to the social and community psychology in Latin-America and Colombia as a result of dialectic processes in which are submitted to natural procedures that are given by the nature or those people and communities that have been already built. Likewise, in this historical background is made reference to ethical-political perspective that have been acquired by social and community psychology in favor of vulnerable sectors and people socially excluded. This matter has achieved to determine priorities of some relevant and outstanding psychosocial processes; such as social inclusion, citizenship construction and community strengthening as powerful personal and community transformation throughout of their own social actors.
\end{abstract}

22 Psicólogo. Con experiencia en la creación, diseño, gestión y desarrollo de proyectos educativos y sociales que promueven la participación, la autogestión y creación de consciencia crítica en población vulnerable. Experticia sistematización de experiencias comunitarias. CvLAC: nombre del grupo investigativo Interdisciplinariedad y prácticas comunitarias. Título de la investigación: Experiencias comunitarias significativas en relación con la psicología comunitaria: huellas de una disciplina en la vida colectiva. Responsabilidad: investigador principal. Año 2008. 
Keys words: social and community psychology, social inclusion, citizenship construction, community strengthening.

Recibido: 10 de diembre de 2008

Aceptado: 5 de abril de 2009

\section{INTRODUCCIÓN}

Si repasamos las leyes fundamentales de la dialéctica materialista, veremos que cuanto existe en el universo se encuentra en continuo y perpetuo cambio y transformación debido a las tensiones y contradicciones que se generan al interior de cada sistema. ${ }^{23}$ Por supuesto que la realidad social no escapa a este movimiento trasformador, incluida en ella el cuerpo científico (paradigmático, teórico y metodológico) quien posee su propio situado histórico, es decir, que ha sido el resultado de un proceso generado por las personas bajo unas circunstancias específicas.

¿Ello qué significa? Pues que esa realidad que objetivamos, que se hace "visible" ante nuestra percepción no ha estado ahí por siempre, que no es producto de fuerzas supranaturales, que no es independiente de nosotros. Es más, que no permanecerá inalterable por toda la eternidad, que siempre estará en continuos cambios así no seamos conscientes de ello, o por el contrario nos opongamos férreamente a sus transformaciones

A las ciencias sociales se les demanda una postura, en lo concerniente a su postura y accionar ético-político diferente a la tradición científica frente a los grandes desequilibrios e injusticias que aquejan a la inmensa mayoría de nuestros pueblos. Este llamado ha resonado en muchas conciencias críticas de científicos que le han apostado a investigar e intervenir con los sectores vulnerables y excluidos de nuestra sociedad.

Convocatoria urgente y perentoria atendida por Orlando Fals Borda ${ }^{24}$ a través de una postura epistemológica, teórica, metodológica, ético-política denominada InvestigaciónAcción Participativa que pone en el centro del dominio de los procesos transformadores a la comunidad misma.

Otro tanto ha ocurrido en el ámbito de la educación de adultos con Paulo Freire ${ }^{25}$ quien pondría a la pedagogía a dialogar con esa nueva realidad. En Pedagogía del oprimido (1970); planteará que en el centro de la praxis de la pedagogía que expone, estará el poder político,

23 La dialéctica materialista es la teoría de la concatenación universal y del desarrollo, y se expresa en tres leyes fundamentales como son: ley de la unidad y lucha de contrarios. Ley de la transformación de los cambios cuantitativos en cualitativos. Ley de la negación de la negación.

24 Orlando Fals Borda, investigador y sociólogo colombiano, nacido en el año 1925 en la ciudad colombiana de Barranquilla. Reconocido como el autor del enfoque metodológico denominado Investigación — Acción — Participativa.

25 Paulo Freire, nació en Recife, Brasil, 1921. Pedagogo. Estudió filosofía en la Universidad de Pernambuco e inició su labor como profesor de historia y filosofía de la educación en la Universidad de Recife. En 1947 comenzó sus esfuerzos para llevar a cabo la alfabetización de adultos, que durante los años sesenta llevó a la práctica en el nordeste de Brasil, donde existía un elevado índice de analfabetismo. 
por tanto, se busca que los oprimidos asuman conciencia de su realidad y se comprometan en la práctica con su trasformación.

Asimismo la psicología social comunitaria ingresaría en este proceso dialéctico en América Latina formando una corriente de pensamiento identitario con apuestas influenciadas por los movimientos de la teología de la liberación que permitió ir constituyendo una mirada propia de su quehacer a favor de las comunidades vulnerables; proceso en el que han contribuido entre otros investigadores Martín-Baró ${ }^{26}$ y Maritza Montero. ${ }^{27}$

La anterior afirmación respecto a los procesos identitarios de la psicología social comunitaria lleva a pensar, entonces, que la psicología no es o no ha sido una, que no ha tenido y mantenido una coherencia o por lo menos una univocidad epistemológica, teórica y metodológica. Así es, las lógicas respecto al quehacer, cómo hacer y con quién se ha movilizado en la historia de esta joven ciencia generando importantes crisis. Ha fijado una posición y un posicionamiento respecto a su actuación o atención preferencial hacia los sectores económicamente menos favorecidos de nuestra sociedad, a las comunidades que se encuentran en estado de exclusión social, privadas del ejercicio pleno de la ciudadanía.

Muy seguramente dichas intervenciones en tales comunidades han dejado alguna huella, evidencias que se pueden rastrear, impactos sociales de relevancia. Experiencias significativas de la psicología social comunitaria que valen la pena ser ubicadas, indagadas e investigadas. Prácticas exitosas de la psicología social comunitaria en Colombia a las cuales debemos acercarnos con el fin de detectar sus posiciones paradigmáticas, las producciones teóricas, los procesos metodológicos y conocer sus impactos sociales transformadores de las personas, grupos sociales y comunidades.

No constituye el interés del presente artículo ser el resultado de tal indagación, ello será objeto de estudio de un próximo capítulo que se desprenderá de este primer escrito. Por el momento, lo que nos interesa es ver los situados históricos tanto de la psicología social comunitaria latinoamericana como de la psicología social comunitaria en Colombia, no con la finalidad de historiar, sino de conocer la dialéctica en que se han movilizado, lo cual ayudará a comprender sus desarrollos epistemológicos, teóricos y metodológicos como sus posturas ético - políticas. Veremos, no las prácticas, sino algunas temáticas que ha venido abordando la psicología social comunitaria con urgencia y que junto con otras disciplinas han logrado establecer y desarrollar unas teorizaciones que partiendo de la realidad social vuelven a ella para dinamizar nuevas prácticas de transformación social.

26 Ignacio Martín-Baró. Nació el 7 de noviembre de 1942, en Valladolid, España. Sacerdote y psicólogo social comprometido con los sectores marginales de América Latina. Contribuyó con el desarrollo teórico de la psicología social comunitaria. Asesinado por el régimen Salvadoreño.

27 Maritza Montero: es licenciada en psicología por la Universidad Central de Venezuela y Máster en Psicología por la Universidad Simón Bolivar. Se doctoró en Ciencias Sociales en la Escuela de Altos Estudios en Ciencias sociales de la Universidad de París. Ejerció su magisterio en la Universidad Central de Venezuela, hasta su jubilación. 


\section{Situado histórico de la Psicología Social Comunitaria}

Wiesenfeld, E. y Sánchez, E. (1991:40) dirán que la Psicología Social Comunitaria es una especialidad de origen reciente, (Estados Unidos hacia 1965), como resultado del cuestionamiento de psicólogos al paradigma vigente en su limitación de considerar y abordar aspectos como la salud mental y la injusticia social.

Newbrough (1992:7) señala tres etapas en las cuales se ha desarrollado la psicología social comunitaria. La primera, considerada como su nacimiento, parte desde 1965 en la conferencia de Swampscott donde se dan sus primeras definiciones y el desarrollo de entrenamiento del psicólogo comunitario. La segunda, comienza con la conferencia de Austin y va de 1975 a 1989, se define su actuar dentro de un ámbito preventivo en la salud mental comunitaria, con una orientación de enfoque ecológico y donde se abordan problemas sociales que padecen los grupos marginados socialmente. Una tercera etapa tiene mayor consideración por los problemas sociales, su abordaje interdisciplinario y la integración del conocimiento generado en estas intervenciones.

Si bien la psicología social comunitaria pareciera partir de un mismo tronco común, sus desarrollos posteriores no lo serían pues las condiciones específicas de países como los de Latinoamérica, debido a su realidad (social, económica, política, histórica), demandarían una nueva postura de pensarse y de actuación a la psicología, lo cual implicaba asumir paradigmas diferenciadores que le fuesen dando su propio corpus epistemológico, teórico y metodológico que se ajustara a la realidad social de los sectores empobrecidos de esta parte del continente.

Autores como Escovar (1980); Marín (1980); Montero (1984); Serrano García y otros (1992) afirman que existen, dentro de la Psicología Comunitaria latinoamericana, diferentes paradigmas: la Psicología Transcultural, la Psicología Social Aplicada, la Psicología Materialista Dialéctica, la Tecnología Social, laPsicologíaSocial para elDesarrollo Social y Económico, la Psicología Social Comunitaria, la Psicología Comunitaria, la Psico-comunidad, entre otros. Dirán que asimismo la psicología social comunitaria ha echado mano de aportes venidos de las perspectivas críticas, educativas como la de Paulo Freire, el mismo socioconstruccionismo, la hermenéutica, el movimiento de la teología de la liberación. Además, dirigen las miradas hacia otras disciplinas científicas como la sociología, la antropología, la lingüística, la comunicación. Esto tendría implicaciones profundas pues sería dar un vuelco fundamental ante el resquebrajamiento de la "fe positivista".

Se reconoce en esta nueva postura de la Psicología Social Comunitaria que tanto los fenómenos y procesos psicosociales como aquello que se elabora acerca de ellos están marcados por la historicidad. Montero (1994: 27/35) planteará que el objeto de la psicología ha de entenderse a partir de su carácter histórico, del devenir de la realidad social, en su constitución por hechos culturales y anclados espacialmente. 
Por su parte, Vásquez (1998:68) dice con respecto a la interpretación de los fenómenos sociales:

\begin{abstract}
"Interpretar un fenómeno social" implica explicar sus condiciones de constitución. Todo fenómeno social es depositario de memoria ya que está conformado en virtud de las relaciones sociales que lo han posibilitado, no sólo en cuanto a su correspondencia con un determinado periodo histórico por las alteraciones producidas por el tiempo (...) Aceptar este planteamiento implica cuestionar la objetividad de los saberes psicosociales al restituirles su dimensión social (Gergen, 1982; Ibáñez, 1989) y no abandonarlos a la hipotética certidumbre de los hechos, que se presuponen como invariables, estables y no sometidos a ninguna contingencia".
\end{abstract}

Para nada se trata de leer la realidad social de una manera lineal pues el reconocimiento de su dimensión histórica significa que tiene un carácter procesual (Ibáñez 1989; Gergen 1973) cuyas implicaciones van mucho más allá de simples intervalos temporales. Ibáñez (1994:229) especificará que el reconocimiento histórico de la naturaleza de lo social no se reduce a reconocer que las sociedades tienen su propia historia, sino que tienen unas implicaciones profundas que afectan tanto al plano ontológico como al plano epistemológico.

El carácter histórico y procesual de la realidad social indica e implica que todo acto o acontecimiento social está históricamente situado, es decir, que ha tenido un momento de inicio debido a causas o factores específicos. Que así aparezcan ante nuestra percepción y elaboraciones mentales, emocionales, imaginativas, mitológicas, representacionales que han estado allí por siempre, hacen parte de esa finitud procesual; incluidos los seres humanos mismos quienes son socialmente construidos (Berguer y Luckman, 1967. Mead, 194).

Argumento igualmente aplicable y válido para la realidad psicológica pues a decir de Ibáñez (1994:276) los objetos que la componen la realidad psicológica de ninguna manera proceden de algo connatural o de la naturaleza humana, o que simplemente son naturales pues no implica en ellos creación alguna; por el contrario son resultados de las prácticas de objetivación que las personas mismas han desarrollado.

Por tanto se ha entender que toda "verdad" por verdadera que parezca ha de ser sometida al cuestionamiento, a la mirada crítica. Un mismo hecho social podrá no solo ser visto sino interpretado, calificado y asumido dependiendo, por ejemplo, de elementos culturales. Ello lleva a Gergen (1999:221) a sostener que:

\footnotetext{
"lo que es 'obviamente verdad y bueno' para una comunidad es frecuentemente fraudulento o moralmente repugnante para otra. En este sentido el construccionismo invita a una continua postura de autoreflexión -incluso de mirar hacia uno mismo. Cada palabra, proposición o propuesta debe ser provisional, abierta a la deconstrucción y a la evaluación político-moral"
}

En esa construcción de la realidad social actúan con fuerza decisoria los elementos lingüísticos. El lenguaje no sólo expresa sino que construye el mundo; Shotter $(1987,1993)$ por tanto, el lenguaje posee tanto funciones descriptivas como formativas. Lo cual lleva a que el sentido formativo del lenguaje implica, entre otras cosa, que la concepción del mundo no se origina 
de la realidad objetiva, sino que "aparece" o se construye a partir del entramado de relaciones que en la cotidianidad mantienen las personas.

Ahora, que esa realidad social parezca que siempre estuvo allí se debe al proceso de naturalización propia de los individuos como forma de anclar su realidad. Sin embargo, cuando se nace, se hace a un mundo ya dispuesto para esa persona, a una cultura con sus relaciones, significaciones, simbolizaciones, emocionalidades y hasta roles socialmente asignados; y es justamente en ese intercambio de lo lingüístico cuando interiorizamos y naturalizamos ese mundo de la vida.

La realidad está para ser conocida y ese conocimiento, incluido el conocimiento científico, es resultado de la creación colectiva. Al respecto Gadamer (1960) e Ibáñez (1990) apuntan que todo el saber de las ciencias naturales, descansa sobre presupuestos hermenéuticos y sobre las interpretaciones inherentes al lenguaje. Es a partir de las prácticas de lo cotidiano como construimos los saberes, la concepción del mundo, los conocimientos sobre nosotros mismos. De esas subjetividades e intersubjetividades, de las prácticas lingüísticas resultan los saberes cotidianos y especializados, es de la realidad social que se fabrican las teorizaciones y no al contrario. Ibáñez (1994:107) dice justamente:

\footnotetext{
"En definitiva, el conocimiento científico tiene en común con el conocimiento sin otras adjetivaciones el hecho de nacer en el seno de la interacción social y de constituirse en el espacio de la intersubjetividad con base en las convenciones lingüísticas, a los presupuestos compartidos y a los diversos procedimientos para establecer un consenso que sólo es posible gracias a la existencia de un mundo de significados comunes. En consonancia con las aportaciones de la teoría crítica, el construccionismo destaca los diferentes intereses que guían las diversas racionalidades científicas y concluye a la inevitabilidad de que los conocimientos conlleven operaciones normativas y repercutan sobre la propia realidad social"
}

Se insiste en que la realidad es una construcción social pues ello tiene unas implicaciones fuertes tanto éticas como políticas. Es decir, que si la realidad social fuese un hecho naturalmente establecido en la vida de los individuos estos estarían condenados simplemente vivir en su círculo de amarguras o gozar perpetuamente de los "dones" económicos que por algún destino darwiniano el azar les proveyó. Así es que, por el contrario, la construcción social se encuentra enmarcada o si se quiere, regida por las leyes de la dialéctica cuyos cambios y desarrollos estarán en continua actividad. Tomás Ibáñez (1996:67) dice algo bastante interesante:

\footnotetext{
“...una construcción social no participa de la metáfora arquitectónica de un edificio que, una vez construido, se mantiene por sí solo. Lo socialmente construido no sólo ha sido construido por determinadas prácticas sociales, sino que esas prácticas lo mantienen de forma dinámica, incesantemente. Si cesan las prácticas, la construcción se esfuma”
}

En necesario entender que esas prácticas sociales que se dan entre las personas. Los grupos sociales crean a su vez instituciones sociales que necesariamente inciden e influencian las prácticas. A su vez, al interior de esas instituciones sociales se crean normas que constriñen y 
condicionan las interacciones sociales, lo cual lleva a que dependiendo de las instituciones, de su formación, de su dinamicidad política se establecen unas relaciones de poder específicas, democráticas unas, excluyentes otras.

Otro elemento epistemológico y teórico que contribuye con la modelación de la Psicología Social Comunitaria es el concepto de reflexividad como elemento crucial en la producción de conocimiento y en la transformación de la realidad social. Al respecto dice Potter (1996:286) que: "la reflexividad se refiere a un conjunto de cuestiones que se plantean cuando consideramos a relación existente entre contenido de una investigación y los escritos y las acciones de los investigadores".

El significado de reflexividad ha sido entendido de diversas maneras, así por ejemplo, la Etnometodología considera que tiene que ver con las descripciones de una situación y con su construcción, pues describirla es construirla, así Garfinkel (1967:1) entiende: "que las actividades realizadas por los miembros para producir y manejar las situaciones de su vida organizada de todos los días son idénticas a los procedimientos utilizados para hacer descriptibles dichas situaciones".

Por su parte, la Sociología del Conocimiento Científico plantea que no son sólo los hechos los que se construyen socialmente, sino que también las descripciones de los modos en que se produce el hecho factual, igual que la manera como se describe constituyen construcciones sociales (Ashmore, 1989).

De otro lado, el paradigma construccionista comprende la reflexividad como la capacidad que tienen los seres humanos de "romper la disyunción objeto/sujeto" (Ibáñez, 1994). Capacidad que ha posibilitado a los seres humanos referenciarse como objeto de análisis, y que abre la posibilidad de construir el mundo de los significados compartidos y la intersubjetividad, como condiciones indispensables para la construcción de lo social. Dirá Ibáñez (1994:231):

\footnotetext{
"Verse con los ojos de los demás, verse en los ojos de los demás, anticipar los efectos de sentido, o de otro tipo, que producimos en los demás, entender lo que hacen o lo que pretenden los demás, en definitiva, hacer que seamos inteligibles para los otros y recíprocamente, todo ello sería imposible sin la conciencia de sí mismo que permite la reflexividad. El bucle recursivo que cierra el 'yo cognoscente' sobre el 'yo conocido' constituye en última instancia la condición de posibilidad de lo social"
}

Las reflexiones sobre el quehacer, cómo hacer y con quién hacerlo ha permitido que se constituyan no sólo un cuerpo ontológico, epistemológico y teórico sino también metodológico en la psicología social comunitaria. Se establece un claro límite entre la psicología social tradicional en consideraciones que tienen que ver con el tema de lo psicológico, el significado de la intervención y el rol del psicólogo.

Se encuentra en Peláez, Cañón, Noreña (2007: 191) que la perspectiva construccionista es una lectura psicosocial que va más allá de la visión del yo individual, lo cual significa que se 
reconoce, en cambio, una psicología social de las relaciones. Planteamiento de trascendencia pues como afirman estos autores: "Una psicología así, tiene la competencia de reconocer la interdisciplinariedad, los recursos y talentos más que centrarse o hacer énfasis en el déficit o la psicopatología”.

Se establece una mirada holística ya que se habla que la intervención psicosocial integra a la intervención psicológica con la intervención social, algo en que Martín-Baró (1983) había llamado la atención: la primera, o sea la intervención psicológica centrada en el individuo y la segunda, dirigida a comunidades, organizaciones e instituciones. Afirman estos autores que: "Esta última implica también, cambios importantes desde la racionalidad del interventor en comunidades que han elaborado necesidades, aspiraciones, problemas. Se explicita la comunidad en tanto que, la intervención actual no apunta al individuo sino que en términos de Ayestarán (1993), citado por San Juan (1996), ha ido a contextos sociales más amplios como familia, escuela, vecindad, organizaciones, instituciones, estructuras sociales, económicas y políticas. Considera además que la intervención psicosocial necesita un modelo ecológico relacional basado en el análisis de los factores físicos, culturales, económicos y políticos que condicionan la estructuración de las redes sociales las cuales a su vez, condicionan la conducta individual".

Desde una postura construccionista, se trata de cuestionar aquello que se ha naturalizado a través de los diferentes dispositivos en que se han venido desarrollando personas, comunidades e instituciones y que se ha transmitido mediante la cultura, los mitos, las ritualidades, las ideologías, las prácticas sociales e institucionales; que a decir de (Gergen, 1996) se "cuestiona lo incuestionable"; por tanto, se trata de resignificar estas construcciones sociales para construir nuevas miradas, nuevas formas de acción y de relación entre personas, comunidades, organizaciones e instituciones. Ello, por supuesto, permite su visibilización como agentes conscientes de transformación social.

Según estos autores es menester establecer ciertas precisiones y diferenciaciones, por ejemplo, entre lo social, sociedad, comunitario; igual que entre intervención psicológica y psicosocial, pare ello dirán, respecto al primer tema, Peláez, Cañón, Noreña (2007: 191):

\footnotetext{
"Es necesario señalar aquí que el concepto de lo social se diferencia del concepto de sociedad, así lo social es del orden de las relaciones y de lo que genera significados, o que se constituye en significativo, mientras que la sociedad hace alusión a un dispositivo a través del cual los hombres se organizan en conjunto. La intervención social comprende o engloba lo comunitario, sólo que al emerger lo comunitario va tomando distancia de los dispositivos de la sociedad sin desconocer las relaciones. Lo comunitario está ligado entonces a la concreción de los sujetos en acción situada sin desconocer lo social, pero contrastando entonces los dispositivos ideológicos de la sociedad"
}

Con respecto al segundo: "La diferencia con la intervención psicológica estriba en que ésta última enfatiza la exploración de aspectos intrapsíquicos, sin apostarle a la conexión de sentido entre lo social y lo individual. Digamos que lo psicosocial pretende integrar la 
dualidad sujeto y sociedad, relación permanentemente abordada por la Psicología social". Según Hernández (2003) citado por Peláez, Cañón, Noreña (2007: 191):

\begin{abstract}
"Agentes de intervención y consultantes forman en conjunto un nuevo sistema que regula la gestión o dirección de sus miembros. La intervención psicosocial se sitúa en la relación que se establece entre categorías sociales y psicológicas. Las categorías sociales sería el objeto sobre el que se interviene, ya se trate de sistemas o estructuras sociales. El efecto inmediato que se busca es el cambio social. El objetivo final sería el cambio personal. Se interviene sobre sistemas y estructuras sociales para resolver problemas psicológicos (objetivo final) a través del cambio social (efecto inmediato). Esto se puede aplicar tanto en la psicología social - comunitaria como a la psicología de las organizaciones y al análisis institucional".
\end{abstract}

En lo referente al para qué hacerlo y con quién hacerlo, es decir sobre las intencionalidades el mismo Hernández (2003) dice que:

"En términos más amplios se trata no sólo de hacer acciones en la comunidad, sino que las personas identifiquen los problemas emocionales, relacionales, sociales y económicos en un proceso de reflexión personal y grupal y que a través de la reflexión - acción se contribuya al cambio individual y social.

La acción de este concepto va orientado a la búsqueda de la autonomía de los grupos y personas involucradas en las alternativas de solución a las diferentes problemáticas, donde la categoría psicosocial juega un papel importante pues articula todas las áreas hacia un contenido más social y más humano, donde las relaciones humanas entre los integrantes de un proyecto, su relación familiar, relación de grupos, relación de parejas, relación con la comunidad y otros".

Para Maritza Montero el objeto de la Psicología Social Comunitaria es el "estudio de los factores psicosociales que permiten desarrollar, fomentar, mantener el control y poder que los individuos pueden ejercer sobre su ambiente individual y social, para solucionar problemas que los aquejan y lograr cambios en esos ambientes y en la estructura social" (Ferullo, 2000: 47). En cuanto al rol profesional el eje común pasa por conceptualizar al mismo en tanto que investigador, tecnólogo, interventor/investigador, catalizador, facilitador y agente de cambio social a la vez que en señalar las implicancias sociales, ideológicas y políticas de dicho rol profesional.

\title{
Situado histórico de la Psicología Social Comunitaria en Colombia
}

Pretender decir que la Psicología Social Comunitaria nace en Colombia como un hecho aislado del contexto latinoamericano, particular y más bien antojoso por parte de algunas psicólogos, es pretender afirmar que los seres humanos no hemos sido producto y no hacemos parte de esas fuerzas que rigen el universo y que se encuentra en permanente interconexión y afectación. A pesar de no darse los fenómenos sociales, científicos, políticos, económicos uniformemente en América Latina, se van relacionando tanto sincrónica como asincrónicamente. En el caso de la Psicología Social Comunitaria en Colombia hace parte del proceso que le dio vida a la psicología social comunitaria latinoamericana, así su desarrollo haya sido temporalmente 
distinto como ciencia. Podríamos afirmar que nace con la Psicología Social Comunitaria latinoamericana, con las mismas miradas y posturas que dieron nacimiento a esta y de la cual se tienen mayores registros.

Si consultamos la literatura que hay sobre el situado histórico de la Psicología Social Comunitaria colombiana, encontramos que no hay muchos registros sobre esta. Carlos Arango Cálad en su ensayo sobre la Historia de la Psicología Comunitaria en Colombia apunta que aparte de los datos recogidos hasta 1995 por Arango (1995) en el capítulo sobre el rol del psicólogo comunitario, de esa fecha en adelante no encuentra publicaciones que den cuenta sobre esta historia. Se da una falta de reconocimiento de todas aquellas experiencias e investigaciones que en el área de la psicología comunitaria han venido realizando psicólogos y psicólogas en el país. Apunta que se conoce mucho más el trabajo realizado en los eventos internacionales que en la misma Colombia.

Lo que se puede detectar con el surgimiento de la Psicología Social Comunitaria es que se producen acontecimientos en la realidad social que marcan el camino para que surjan o bien se instalen, en nuestro caso colombiano, disciplinas científicas como la sociología y la psicología. Según Arango (2005) dos hechos significativos se produjeron en 1948 tanto para la comunidad colombiana como en la historia de la psicología como fueron: de un lado, el nacimiento del primer programa de psicología en el Instituto de Psicología Aplicada de la Universidad Nacional de Colombia; de otra parte, el asesinato de Jorge Eliécer Gaitán que recrudece la guerra fratricida y que ha sido conocida "La violencia en Colombia" y el desarrollo de procesos comunitarios que dieron paso a que comenzara a fundamentarse la Investigación -Acción - Participativa de Orlando Fals Borda en su intervención con los campesinos de los Andes y el método que denominó "acción comunal".

En la década de los sesenta la realidad social mundial sería de gran agitación, lo que necesariamente incidiría en diferentes países de América en aspectos como: la ruptura de las ciencias sociales con el establecimiento formal académico; en la influencia propiciada en los movimientos sociales; en las políticas gubernamentales; en las subjetividades de las personas. En esta década se daría la Revolución Cubana; surgirían movimientos guerrilleros en varios países de la América Latina; se implantaría el programa "Alianza para el Progreso desde la OEA como intento de paliar los problemas del subdesarrollo de esta región; la guerra de Vietnam traería una movilización por los derechos civiles, lucha contra la pobreza, las expresiones pacifista del movimiento Hippie; la revolución de "Mayo del 68" encabezada por intelectuales y jóvenes; surgirían los movimientos pacifistas, ecologistas, feministas y de las minorías sexuales; aparecería en el ámbito disciplinar lo que se ha conocido como la antipsiquiatría; lo mismo la aparición de la psicología comunitaria como intención de neutralizar los movimientos sociales.

Como habíamos referenciado al principio de este artículo, Paulo Freire tendría su cuota de contribución en este panorama aciago de los sectores populares con sus propuestas y prácticas en la Educación de Adultos. Pondría igualmente su aporte el movimiento denominado "Teología de la Liberación" que no sólo irradiaría su accionar hacia la población socialmente excluida, 
sino que influenciaría las posturas de la Psicología Social Comunitaria Latinoamericana con dos conceptos fundamentales como el de horizontalidad e historicidad, amén de las posturas ético - políticas que este movimiento venía propugnando a favor de los sectores populares.

Colombia, con sus propias características internas: sociales, políticas, narcotráfico, genocidios, desplazamiento forzado de la población, concentración de la riqueza; no será por supuesto ajena a lo que ocurría en el ámbito mundial en esta década. Como no lo sería la psicología, que enfrentaría la discusión y cuestionamiento de su abordaje e intervención exclusivamente individualista. Otro tanto ocurriría en las ciencias sociales que pondrían en crisis los paradigmas hegemónicos y que en el caso de Orlando Fals Borda, por la misma estrechez de mirada y accionar de la academia, la abandonaría y dedicaría sus esfuerzos a trabajar y acompañar procesos comunitarios. Se trataba de implementar esa nueva mirada que tenían algunos investigadores de las ciencias sociales que buscaban reconocer el saber popular de origen comunitario, implementar una nueva metodología en la producción de conocimiento en que se rompía la neutralidad del investigador y la relación de la tradición positivista que suponía la separación entre sujeto - objeto de estudio.

Necesario recordar que para la época en nuestro país las comunidades cristianas de base y las comunidades cristianas campesinas asumirían los planteamientos de la teología de la liberación, la educación liberadora, las técnicas participativas, las dinámicas grupales y el cuestionamiento de la realidad. Tan fuerte fue el cuestionamiento de dicho sector religioso que el capellán de la Universidad Nacional, Camilo Torres Restrepo, encabezaría un movimiento político y terminaría por vincularse a la guerrilla del Ejército de Liberación Nacional, ejemplo que siguieron algunos otros curas que llegaron a hacer parte de la dirección de la insurgencia armada, con los consecuentes desenlaces que se conocen.

Afirma Arango (2005) que tanto la situación mundial como la regional y local serían las bases para que apareciera la Psicología Social Comunitaria en Colombia. Entraría en cuestionamiento el control social que los profesionales de dichas disciplinas ejercían en nombre de la psiquiatría y la psicología, ello llevaría a que se diseñara un nuevo rol y cómo se vincularía con los sectores comunitarios. Algunas experiencias significativas que permitirían la constitución y desarrollo de la psicología social comunitario fueron: el hospital Psiquiátrico de Boyacá que a comienzos de 1970, e inspirado en el movimiento de psiquiatría democrática de Italia y los postulados de Franco Basaglia se convirtió en hospital de puertas abiertas donde los pacientes podían entrar y salir libremente. Con el ejemplo del Hospital Psiquiátrico de Boyacá un grupo de profesores y estudiantes en 1975 constituyó un seminario extraacadémico de Antipsiquiatría de la Universidad Nacional el cual no tenía matrículas, ni exámenes, ni límites de tiempo donde se discutió sobre estos aspectos. Un evento de trascendencia para el desarrollo de la Psicología Social Comunitaria sería el Simposio Mundial de Cartagena sobre Investigación Acción Participativa, pues se legitimaría en la comunidad científica esta metodología.

Como parte del panorama de la historia de la Psicología Social Comunitaria en Colombia referenciaremos que desde la década de los 60's en adelante comenzaría a aparecer en las 
universidades nuevos programas de psicología, uno de ellos en los 70's con enfoque comunitario como fue el de la Universidad del Valle. Dirá Arango (2005) que en los 80's la psicología comunitaria tendría un reconocimiento público expresado en: La reflexión epistemológica y teórica que se debatieron en eventos profesionales y científicos en psicología comunitaria. La catástrofe de Armero que confrontó a los psicólogos de país con sus modelos tradicionales que no daban respuestas a las necesidades del momento como eran las de constituir tejido social, elaboración de duelo, generación de ingresos, organización comunitaria, generación de proyectos; ello cuestionó el tipo de formación profesional que se impartía en la academia Colombiana en el área de la psicología (Mejía Barrero y Jiménez, 1990). Así mismo dentro de ese reconocimiento surgieron nuevos programas de psicología comunitaria. Otro hecho que contribuyó con esta finalidad, aunque lamentable, fue el asesinato del psicólogo y sacerdote jesuita Ignacio Martín-Baró quien cuestionara la psicología tradicional y constituyera lo que se conoce como la psicología crítica que comprometía a la psicología con dilucidar las causas de injusticia, pobreza y violencia y trabajar por cambios radicales en esa realidad latinoamericana.

Considera Arango (2005) que otro de los hitos en el desarrollo de la disciplina se trata del surgimiento del programa en pregrado en Psicología Social Comunitaria (1995) de la Universidad Nacional Abierta y a Distancia (UNAD) que respondía a las necesidades del momento, aún vigentes en nuestro país, de formar profesionales que acompañaran a las comunidades en la solución de su problemática psicosocial, económica, de fortalecimiento comunitario, de inclusión social, de convertirse las comunidades en los artífices de sus propios cambios y transformaciones; un psicólogo y una disciplina que leyera la compleja realidad Colombiana desde un quehacer diferente al tradicional de la disciplina. Programa que ha colaborado decididamente con la transformación de las comunidades vulnerables, y que ha venido actuando en los más apartados rincones del país así como con las más diversas comunidades y culturas.

En lo corrido del programa de psicología la UNAD ha formado hasta el momento más de 10.000 psicólogos social comunitarios en los diferentes CAED del país. Dice Arango (2005) que el $89.66 \%$ de estos egresados se desempeñan en actividades que tienen que ver con lo comunitario. Infortunadamente a finales de 2005 y bajo los criterios de la Asociación Colombiana de Facultades de Psicología - ASCOFAPSI - el programa desapareció como tal, pues promueve esta asociación una política oficial de formación básica en psicología en pregrado, dejando la formación comunitaria en psicología como un campo de especialización. Hecho que para nada favorece a quienes han de ser los depositarios directos de las acciones de los profesionales de la psicología social comunitaria como son los miles de Colombianos que se encuentran en situación de desprotección social, económica, política y que en los últimos años con el incremento de la guerra, los genocidios, los desplazamientos forzados que no solo se han incrementado en número sino que ha complejizado aún más esta situación catastrófica de Colombia. Sin embargo, queda una pequeña luz de esperanza en el camino, pues conforme a los principios rectorales de la UNAD de privilegiar la proyección de la universidad hacia los sectores vulnerables se ha mantenido el énfasis comunitario en este pregrado. 
Como se ha podido ver estamos en mora de sistematizarse la historia de la Psicología Social Comunitaria de Colombia, lo mismo que las prácticas exitosas que indudablemente ha tenido en su acompañamiento a las comunidades tanto de los grandes centros urbanos como de las más apartadas regiones de nuestro país. Así mismo hay todavía camino por recorrer en la relación dialéctica con los centros de poder que imponen los contenidos y orientaciones de los programas académicos de la psicología y el rol del psicólogo con el prurito muchas veces de la globalización. Sin embargo y pese a las posturas paradigmáticas de la tradición positivista y de los anclajes en el que hacer de la psicología lo que encontramos es que la Psicología Social Comunitaria en América Latina ha venido adelantando intervenciones a partir de múltiples procesos psicosociales; algunos de ellos tienen que ver con la inclusión social, la construcción de ciudadanía y el fortalecimiento comunitario, que de la praxis misma han permitido generar teorizaciones importantes de las cuales daremos someramente cuenta en los siguientes renglones.

\section{Inclusión social, construcción de ciudadanía y fortalecimiento comunitario, como perspectiva de intervención de la psicología social comunitaria latinoamericana}

Tal vez podría afirmarse que la dinamización de estos procesos psicosociales no son experiencias exitosas exclusivas de la psicología social comunitaria, ya que podemos evidenciar que otras disciplinas y prácticas comunitarias, como la educación de adultos a través de la alfabetización, las han venido implementando como parte del presupuesto de transformaciones de personas y comunidades.

Aún no siendo de autoría exclusiva de la Psicología Social Comunitaria esta disciplina científica ha contribuido decididamente con el desarrollo de estas tres temáticas tanto en la praxis como a nivel teórico, lo que ha enriquecido su comprensión epistemológica y teórica como también optimizar su aplicación en las comunidades marginales de la América Latina. Veremos entonces a continuación los presupuestos teóricos de estos tres procesos psicosociales como son la inclusión social, la construcción de ciudadanía y el fortalecimiento comunitario a través de las elaboraciones conceptuales de diversos científicos del pensamiento social.

\section{De la exclusión a la inclusión social}

Para hablar de inclusión social habría que hacerlo primero de la exclusión. Esta última se puede entender como la negación de un sistema social a que sus miembros tengan la oportunidad y posibilidad real de disfrutar de la totalidad de beneficios, bienes y servicios de esa sociedad como los económicos, políticos, culturales, educativos, laborales, salud, espirituales, estéticos, entre otros; promovidas por cuestiones étnica, raza, género, cultura, opción sexual, clase social, pensamiento político e ideológico y religioso, como por su estado de ser una persona con capacidades especiales.

Dice Buvinié (2004) que por su misma amplitud el concepto de exclusión social puede tener diversos significados, no obstante sobre sus características generales y principales indicadores y la forma en que se relaciona con la pobreza y la desigualdad existe consenso (Sen, 2000; 
Bourguignon, 1999; Rodger, Gore y Figueiredo, 1995). Además de la insuficiencia en los ingresos se refiere a un conjunto más amplio de circunstancia que la pobreza.

"La exclusión social está más estrechamente relacionada con el concepto de pobreza relativa que con el de pobreza absoluta, y por tanto, indisolublemente vinculada con la desigualdad. Se refiere no solo a la distribución del ingreso y los activos (tal y como lo hace el análisis de pobreza), sino también a las privaciones sociales y a la falta de voz y poder en la sociedad". Buvinié (2004)

Para Tsakloglou y Papadopulus (2001) la exclusión social hace referencia a la incapacidad del individuo de participar en el funcionamiento básico político, social y económico de la sociedad en que vive. En tanto que para Behrman, Gaviria y Székely (2003) se trata de la negación del acceso igualitario a las oportunidades que determinados grupos de la sociedad imponen a otros.

La exclusión social tiene un situado histórico y unas causas que la producen, no es el resultado de designio de divinidad alguna o mano sobre natural, es producto de la relación que se produce entre los seres humanos. Para Aggleton, Parker y Maluwa (2002) la exclusión social no es producto del azar sino que es resultado de las relaciones sociales que establecen los individuos; es arbitraria pues las personas son excluidas debido a características adscritas antes que adquiridas y que están al margen de la agencia o responsabilidad del individuo. Entre los estudiosos del tema se está de acuerdo en que la exclusión social tiene dimensiones tanto espaciales como intergeneracionales, con unas características de permanencia antes que de transitoriedad, Gray - Molina, Pérez de Rada y Sojo (2003).

\section{Características comunes de la población excluida}

La exclusión social en América Latina posee un acumulado histórico de terribles injusticias que permitió la prosperidad de unos pocos en contra de la gran mayoría de la población. Respecto a sus orígenes en esta parte del hemisferio Buvinié (2004) anota:

"La exclusión social está tallada en la historia de América Latina. Es producto de la explotación colonial de los recursos y habitantes autóctonos, incluida la trata de esclavos africanos y el trabajo forzado de los pueblos indígenas. Y es producto de décadas, o bien de siglos, de persiste desigualdad. Las poblaciones con una historia de exclusión tiene historias, identidades sociales y agendas diferentes, y el origen de la exclusión es único. Sin embargo, todas ellas componen características y mecanismos comunes de exclusión"

Algunas de las características de la exclusión social refieren a que los grupos socialmente exclusión han sido invisibilizados en las estadísticas oficiales. "América Latina sabe más sobre la diversidad de su producción industrial que sobre la diversidad de sus pueblos”, Buvinié (2004). Así mismo soportan los índices más altos de pobreza; por ejemplo, el sector más amplio de la población de América Latina está constituido por pobres; en Bolivia, Guatemala y Perú los grupos étnicos alcanzan el $60 \%$ de quienes viven por debajo de la línea de pobreza (BID, 2003; Patrinos 2000). De otra parte, los sectores excluidos socialmente son 
marcados por la estigmatización y la discriminación. Las mismas relaciones que se establecen con ellos generan prevención y hasta temor, en Colombia. Tal es el caso del habitante de la calle marcado con el epíteto de desechable con todas las connotaciones y consecuencias que ello implica. Al pobre se le atribuye su estado de pobreza debido a la pereza. Link y Phelan (2002) anotan que las diferencias de poder están en la esencia de lo que es el estigma, debido a que los grupos con escaso poder no están en condiciones de estigmatizar a otros. El estigma influye, significativamente en la distribución de oportunidades de vida.

La discriminación, con frecuencia, está atada al estigma y es otra característica compartida por los grupos excluidos. Deutsch, 2002; Patrinos, 2000; Arias, Yamada y Tejerina, 2002; Mezzera, 2002; afirman que:

"La discriminación puede ser resultado de la imposición social o de la "autodiscriminación", en la que la herencia de la discriminación pasada desalienta a los individuos a postularse para determinados empleos, concurrir a centros de salud o de defender sus derechos. En América Latina existe evidencia suficiente de discriminación salarial en el mercado laboral que afecta a las mujeres, a los pueblos indígenas y a los afrodescendiantes".

Ahora bien, cuando se reúnen más de dos de las características anteriormente descritas de exclusión de grupos se presentan desventajas acumulativas. Buvinié (2004) citando un informe de Unaids (2002):

"En Honduras las garífunas, un grupo de afrodescendientes, exhibe uno de los más altos índices de $\mathrm{VIH} / \mathrm{SIDA}$ en la región. El estigma asociado con el VIH/SIDA se basa en los prejuicios de género y los refuerza. Por ello, suele culparse más a las mujeres que a los hombres por padecer VIH/SIDA y son ellas quienes sufren mayor estigma que su contraparte de sexo masculino".

Tal ha sido la preocupación por este fenómeno social que reiteradamente se ha ventilado en diversos escenarios como: La Cumbre de las Américas celebrada en Quebec en 2001. La Conferencia de la Mujer de las Naciones Unidas (1995): La Cumbre social de las Naciones Unidas (1996). La Conferencia de las Naciones Unidas contra el racismo (2001). Los trabajos de Amartya Sen (1999) referidos a derechos y objetivos de desarrollo. Este cúmulo de preocupaciones ha hecho que la banca multilateral como el Banco Interamericano de Desarrollo y organismos internacionales adopten como meta la inclusión social dentro de sus propósitos y que hayan apoyado investigaciones sobre las causas de la pobreza y la desigualdad y las medidas tendientes a corregir la exclusión social en la región.

Sin embargo, frente a estas preocupaciones podemos decir que en el fondo de algunos de estos organismos, en especial la banca multilateral, puede existir el temor de que llegue un momento en el cual la problemática sea de tal dimensión que desborde la capacidad de respuesta institucional frente a posibles movilizaciones sociales. Lo que se ve es que en término generales las condiciones de vida de los sectores vulnerables no mejoran, e incluso ha comenzado a horadar aquel otro segmento social que tradicionalmente se denominaba la clase media. 
Situación lamentable que padece la mayoría de la población de la América Latina que demanda del sector gubernamental políticas públicas de inclusión social, que permita la visibilidad y el goce de los bienes materiales y culturales a todos las personas que habitan este continente sin distingo de raza, género, religión, condición social. Se requiere por parte de los gobiernos cambios estructurales no políticas asistencialistas, elaborar una agenda, formular unos planes de desarrollo que permitan eliminar las desventajas acumulativas que por siglos han sumido en la pobreza a millones de familias.

Para las comunidades vulneradas en sus derechos sociales, económicos y civiles la situación requiere con urgencia de su agenciamiento comunitario, el fortalecimiento como sociedad civil, de alianzas estratégicas con sectores y organizaciones del ámbito nacional e internacional; además, de una labor de autoreconocimiento de sus capacidades y potencialidades y de acciones organizadas y planeadas en la exigibilidad de sus derechos y por supuesto de su constitución como ciudadanos plenos tanto de deberes como de derechos.

\section{Construcción de ciudadanía}

Inclusión social y construcción de ciudadanía se convierten en dos vertientes inseparables del desarrollo social. No podrá haber real ejercicio de la ciudadanía si prevalecen las condiciones de exclusión social. Entonces, ¿Cómo podría entenderse la ciudadanía?

Nelia Bojórquez (Oficial del Programa de Derechos de la Niñez y la Mujer, unicef México) en su artículo sobre Ciudadanía dice que esta va más allá de percibirse como titular de derechos, pues implica así mismo la participación y el compromiso con el destino de la sociedad. De esta manera, la globalización ha roto la idea tradicional que se tenía sobre ciudadanía en el sentido de pertenencia.

Por su parte T.H. Marshall (1950) asume los derechos civiles, políticos y sociales como estatuto social que determina el sentido de pertenencia a la comunidad nacional y favorece la participación en la vida social. De su lado Vargas (1997:62) dice que la ciudadanía política implica el derecho a elegir y ser elegido, mientras que la ciudadanía social se refiere a la prerrogativa de cada persona de gozar de un estándar mínimo de bienestar económico y seguridad (derecho a la seguridad social, salarios, beneficios sociales, entre otros). Touraine (1992) identifica la ciudadanía con la conciencia de pertenecer a la sociedad y con la capacidad del individuo de sentirse responsable del buen funcionamiento de las instituciones, en simetría con la obligación de las mismas de respetar los derechos de hombres y mujeres.

La UNICEF, siguiendo las posturas tanto de Marshall como de Touraine, plantea que la ciudadanía permite a las personas construir o transformar las leyes que ellas mismas quieren vivir, cumplir y proteger para la dignidad de todos. Concepto de ciudadanía que contempla el ámbito objetivo y subjetivo. El objetivo relacionado con las condiciones externas que garanticen un efectivo cumplimiento del derecho que les asiste a las personas. El subjetivo referido a la habilitación de la persona para ejercer sus derechos. La ciudadanía es definida 
entonces por la participación en la aplicación y la formulación del derecho, con conciencia de causa y en la práctica de su vida.

Por su parte Bustelo (1999:41) habla sobre la construcción de una ciudadanía activa de la siguiente manera:

“... pobres no son solo aquellas víctimas, de una u otra forma, de la mala distribución de ingresos y la riqueza, sino también son aquellas que sus recursos materiales e inmateriales no les permiten cumplir con las demandas y hábitos sociales que como ciudadanos se les exige. Por eso la pobreza es sobre todo, pobreza de ciudadanía. La pobreza de ciudadanía es aquella situación social en la que las personas no pueden obtener las condiciones de vida —material e inmaterial - que les posibilite desempeñar roles, participar plenamente en la vida económica, política y social y entender los códigos culturales para integrarse como miembro de una sociedad. La pobreza de ciudadanía es no pertenecer a una comunidad en calidad de miembros plenos, y esto es, la exclusión social".

Hay dos formas de pensar políticas de ciudadanía, dice Bustelo (1998:244-250) para enfrentar los niveles de desigualdad, vulnerabilidad y exclusión social que se viven en América Latina: la ciudadanía asistida de una parte, y de otra, la ciudadanía emancipada. La primera corresponde al modelo hegemónico y se relaciona con el denominado consenso de Washington que parte de una concepción atomística de la sociedad en la que solo se reconocen individuos con intereses, el mercado aparece como un mecanismo de autorregulación y la presencia del estado será mínima, perspectiva cuya naturaleza de ciudadanía es civil. La ciudadanía política se expresa en derechos formales como el derecho a elegir y ser elegido, y los derechos sociales no son demandables. No existe preocupación alguna por la distribución de la riqueza; y las política sociales son concebidas como focalizadas a los grupos más vulnerables mediante subsidios. Para Bustelo el fundamento del subsidio es la compasión. En el mismo sentido Franco (2003:7) dice que no es casual que los programas sociales estatales estén basados en la oferta y no en demanda; de esta forma, parte de las políticas sociales contribuyen a la manutención de la pobreza y no a su erradicación.

Prosiguiendo con Bustelo (1998:244-250) se entiende la ciudadanía emancipada como una propuesta socialmente inclusiva. El modelo de ciudadanía emancipada se basa en la igualdad social, la cual se entiende como:

"Derecho de las personas —en tanto que miembros de/socios de un esquema de cooperación social común —a tener iguales oportunidades de acceder a beneficios social y económicamente relevantes. Igualdad implica equidad - proporcionalidad en el acceso a los beneficios y costos del desarrollo —y también, justicia redistributiva basada en la solidaridad colectiva"

Vale decir que la construcción de ciudadanía se ha de convertir en una acción solidaria de los propios actores sociales sin ninguna delegatoriedad. Corresponde a hombres y mujeres por igual ser los participantes y beneficiarios directos de estos procesos de inclusión social a través de la constitución de ciudadanía. Se estaría hablando entonces de la autonomía de los ciudadanos para transformar su entorno vital. Habermas (1999:258) citado por Oraisón y Pérez 
(2006:15-29) considera que la autonomía de los ciudadanos no depende ni de las libertades subjetivas ni de los derechos de prestación garantizados para los clientes del Estado de bienestar, sino de asegurar conjuntamente las libertades públicas y privadas. Continúan diciendo al tenor de Habermas que de igual modo, los derechos subjetivos que garantizan la vida autónoma sólo pueden ser formulados adecuadamente cuando los propios afectados participan por sí mismos en las discusiones públicas acerca del contenido de estos derechos, en relación con aquellos asuntos que, directa o indirectamente, los afectan.

En consecuencia Oraisón y Pérez (2006:15-29):

"los procesos de construcción de la ciudadanía se entrelazan con las posibilidades de participación genuina de los involucrados a partir del desarrollo de la propia práctica. Vale decir que, no se trata de un estatus formal a priori, sino de un proyecto de acción emancipadora y transformadora del sujeto y de la realidad que lo constituye".

Ya Freire, P. (2002: 67) en la década de 1960, dicen Oraisón y Pérez (2006:15-29), en el marco del pensamiento de la sociología latinoamericana y la teoría de la dependencia, expresa en textos tales como la Pedagogía de la liberación o La educación como práctica de la libertad, su preocupación por la educación y su vinculación con los procesos de construcción de la ciudadanía. Para este autor, estos procesos constituyen una búsqueda permanente e inacabable de la completud humana, proceso de humanización en el que el hombre se realiza como tal, y de modo específico, a través de procesos crecientes de concientización; esto es, insertándose en la realidad de manera crítica. Así, ser ciudadano es ser sujeto de la historia, de su propia historia, que se construye en primera instancia en la comprensión de la realidad de su sometimiento, deshumanización y negación de la ciudadanía.

La construcción de ciudadanía se encuentra estrechamente ligada con el hecho de la participación, de la solidaridad; remite a la subjetividad del individuo y a la intersubjetividad que se genera entre las personas, son codependientes. En este sentido, apuntan Oraisón y Pérez (2006:15-29) citando a Ferullo, P, (2006:195-196): reconocemos a la participación crítica «en tanto es la que más se adecua como potencial disparador de los efectos potenciadores de los sujetos que se buscan obtener: crecientes niveles de conciencia, de capacidad autogestiva y organizativa, de posibilidad de asumir compromisos y responsabilidades tanto en relación con cuestiones personales como sociales [...] un aprendizaje que brinde a los sujetos la posibilidad de una mayor incidencia deliberada en el rumbo de sus vidas personales y/o comunitarias».

Hernández, E., (1994) ha identificado tres dimensiones fundamentales de una participación comunitaria activa:

- El ser parte: búsqueda referida a la identidad, a la pertenencia de los sujetos.

- El tener parte: referida a la conciencia de los propios deberes y derechos, de las pérdidas y ganancias que están en juego, de lo que se obtiene o no.

- El tomar parte: referida al logro de la realización de acciones concretas. 
Participación como condición fundamental de la acción que se basa en una concepción de igualdad, equidad en la transformación de las personas y de su realidad social. No es ni mucho menos una participación formal que no produce cambios ni transformaciones tanto para el sujeto como para la comunidad y que en cambio puede servir de legitimación del orden socialmente impuesto.

Abordadas, de manera somera, tanto la inclusión social como la construcción de ciudadanía a manera de procesos psicosociales decisivos en la cristalización de la justicia social, habría que pensar en un proceso psicosocial que las potencie, que ofrezca herramientas a las comunidades para convertirse en actores fundamentales de sus propias transformaciones.

\title{
Fortalecimiento Comunitario
}

El fortalecimiento comunitario se convierte en estrategia y herramienta para que personas y comunidades comprendan y reconozcan el carácter histórico de la vida cotidiana, sepan de los factores que la originan, las consecuencias que acarrean esas contradicciones; deconstruyan esa realidad social a partir de una reflexión crítica y se asuman como actores fundamentales del cambio y transformación personal y de su propio entorno.

Es de aclarar que el fortalecimiento comunitario, si bien ya se venía incluyendo en la agenda de las promotorías comunitarias en esta parte del continente hacia los 60 , no es exclusivo del entorno de América Latina. Existen sistematizaciones que dan cuenta de ello y obras como la de Julián Rappaport que en 1981 hablaban sobre empowerment o empoderamiento. Sin embargo, para efectos de este artículo interesa lo planteado por Montero (2007:293-316).

\section{¿Qué es fortalecimiento?}

Montero (2007:295) lo define de la siguiente manera:

\begin{abstract}
"Por fortalecimiento se entiende el proceso mediante el cual los miembros de una comunidad o un grupo - sean ellos miembros de grupos organizados dentro de esa comunidad o personas interesadas en promover y logra un cambio respecto de algunas circunstancias que afecta a esa comunidad o grupo - desarrollan conjuntamente capacidades y recursos para controlar su situación de vida (aquella en la cual están en un momento específico); actuando de manera comprometida, consciente y crítica, para lograr las transformaciones de las condiciones que juzgan negativas o que deben ser modificadas según sus necesidades y aspiraciones, transformándose al mimo tiempo, a sí mismo"
\end{abstract}

El fortalecimiento comunitario, expande la libertad de escoger y de actuar, aumenta la autoridad y el poder del individuo sobre los recursos y las decisiones que afectan su vida. Se trata de verdaderos juegos de poder entre exclusores y excluidos, por obtener los últimos, los derechos plenos que la ciudadanía les confiere por igual a todos. Podría decirse que el fortalecimiento comunitario es una pedagogía del aprender de manera activa, crítica y reflexiva a transformarse en lo personal y social. Se trata de una práctica en la que se asume el poder político que ha estado concentrado, Freire (1970:47-48). 


\section{¿Cuáles son los elementos fundamentales del fortalecimiento?}

Montero (2007:295) apunta a que estos son algunos de esos fundamentos que aparecen en la literatura especializada:

- Control sobre sus propias vidas, los acontecimientos, recursos y logros por parte de personas y comunidades.

- Comprensión crítica del medio ambiente.

- Unión de fortalezas y competencias individuales; sistemas de ayuda y conducta proactiva con políticas de cambio social.

- Intervenciones políticas dirigidas a aumentar el control que las personas pueden ejercer sobre sus vidas.

- Participación y compromiso de las personas afectadas por una circunstancia juzgada y sentida negativamente por ellas.

- Consciencia de las razones, causas y consecuencias de lo que se quiere hacer.

- Poder para ser autogestionarios, haciendo uso de las capacidades individuales y colectivas, y para exigir aquello que como ciudadanos nos corresponde.

- Politización, es decir, capacidad para hacer públicas las demandas y acciones de transformación social al entender que son parte del ser ciudadanos, miembros de la sociedad civil.

- Identidad social en sus diversas expresiones.

Como se nota, se trata de verdaderos procesos políticos donde la comunidad asume como propia, sin delegatoriedad de ninguna especie, la exigibilidad de sus derechos, por tanto, se puede afirmar que se convierte en sujeto de derecho, lo más importante que se reconoce como tal y se visibiliza en el mundo de las instituciones y del estado.

\section{¿Qué valores sustentan el proceso de fortalecimiento?}

Montero (2007:296) establece cuatro valores fundamentales:

- Autodeterminación como la capacidad que debe tener y disfrutar una comunidad para elegir un destino, para cambiarlo y pare decidir cómo llevarlo.

- Justicia distributiva en la que el fortalecimiento deberá llegar a todos los participantes e incluso a quienes no han participado, pero podrían hacerlo en un futuro ya que son parte de las oportunidades.

- Participación democrática que consiste en la oportunidad para toda persona de la comunidad de participar en la medida de sus capacidades, comprensión y deseo, respetando e incluyendo la diversidad de y en quienes integran esa comunidad.

- Colaboración como valor que inspira el carácter relacional del fortalecimiento comunitario. Se trata de la colaboración mutua que se da entre los miembros de una comunidad. 
Podría pensarse de manera un tanto ingenua que el fortalecimiento comunitario se da de manera sincrónica en las personas y la comunidad y que una vez instalado en ellos permanecerá inmodificable, lo cual no es así. A partir de sus prácticas con comunidades, Montero (2007:298) apunta:

"El fortalecimiento es un proceso complejo que ocurre tanto en el nivel individual como en el nivel colectivo simultáneamente y también separadamente. Esto significa que el grado de fortalecimiento no es homogéneo para todos los miembros de una comunidad. Tampoco se da de una vez para siempre. Como proceso debe estar siempre presente en el trabajo comunitario"

\section{Componentes intrapersonales del fortalecimiento comunitario}

Conforme a Montero (2007:297-298) el componente intrapersonal deriva de la autopercepción que puedan desarrollar las personas participantes de algún proyecto comunitario (agentes externos y agentes internos), respecto de su propia capacidad de influir en aquellos sistemas e instituciones sociales y políticas a los cuales atribuimos, social e individualmente, importancia.

- El control personal que los participantes en proyectos comunitarios puedan desarrollar sobre su autoeficacia al manejar y controlar sus capacidades personales en función de objetivos deseados.

- El desarrollo de creencias positivas y realista sobre su capacidad personal de influir sobre sistemas e instituciones sociales y políticas.

- La historia personal y grupal relativo al ejercicio de dicha influencia, razón por la cual es tan importante planificar el trabajo comunitario de manera que quienes participan puedan obtener logros concretos como efecto de sus acciones.

- Los sentimientos de apego a la comunidad y de pertenencia e identidad respecto a ella.

- Consciencia crítica sobre las condiciones de vida.

\section{Componentes comportamentales del fortalecimiento comunitario}

La misma autora define este componente como las acciones de carácter participativo que se realizan para influir sobre los sistemas sociales y políticos del entorno en el cual se realiza un proyecto comunitario. Así mismo señala cuatro componentes como son:

- Control de personas y grupos de la comunidad sobre el proyecto que adelantan; el cual ha de establecerse durante todo el proceso de planificación, discusión y ejecución.

- Irradiar organizaciones dentro de una comunidad, se trata de construir una variedad de organizaciones al interior de esta.

- Fomento de la participación dentro de la organización, rotación de los liderazgos, lo cual genera compromiso y responsabilidad.

- Producir intervenciones significativas para la comunidad a partir de la construcción, desarrollo e implementación participativa de recursos adecuados. 


\section{Componentes relacionales del fortalecimiento comunitario}

Montero (2007:299) los define como las transacciones que se pueden dar entre las personas participantes y el contexto en que viven mediante las cuales pueden intervenir e influir exitosamente sobre los sistemas sociales y políticos. Que se hayan presentado estos componentes por separado no significa que se hayan de dar de la misma manera en la comunidad. Cinco son los componentes relacionales que distingue la autora:

- Para alcanzar las metas fijadas en el trabajo comunitario es necesario el conocimiento compartido, la disponibilidad y manejo de recursos compartidos. Se habla de recursos entendidos como materiales e inmateriales.

- Para alcanzar los objetivos con la transformación propuesta se requiere del compromiso individual y colectivo.

- Capacidad para relacionar reflexión y acción, traduciendo las ideas en acciones productivas y produciendo ideas creativas a partir de la acción.

- Desarrollo de capacidad para dirigir, tomar decisiones y solucionar problemas.

- Desarrollar experiencias en el trabajo asociativo, colectivo y de colaboración; de tal manera que multipliquen y enriquezcan las capacidades para construir y para generar formas de creación y de transformación colectivas.

Estamos de acuerdo con Montero cuando plantea que de ninguna manera se puede pensar que se trata de una fórmula con el objeto de ser implementada en la comunidad como si fuese algo mecánico y respondiera a meros estímulos y respuestas. La complejidad del mundo de las personas y las comunidades no funcionan de esa manera. Si bien se han esbozado aspectos fundamentales del proceso de fortalecimiento comunitario, también es cierto que se requiere de toda la creatividad, disposición, compromiso y humildad por parte del agente externo para su implementación.

\section{CONCLUSIONES}

A lo largo de este artículo se realizó un sucinto recorrido por el situado histórico de la Psicología Social Comunitaria tanto en Latinoamérica como en Colombia. Asimismo se abordaron tres procesos psicosociales como son inclusión social, construcción de ciudadanía y fortalecimiento comunitario a partir de los cuales esta disciplina ha venido contribuyendo con las comunidades vulnerables en la transformación de su realidad social. De este escrito se desprenden algunas conclusiones como:

- Inclusión social, construcción de ciudadanía y fortalecimiento comunitario son tres procesos psicosociales fuertes con los cuales la Psicología Social Comunitaria ha venido potenciando dinámicas trasformadoras en las comunidades vulnerables.

- Elquehacerde la Psicología Social Comunitaria Latinoamericana en el acompañamiento y postura ético - política a favor de los sectores económicamente deprimidos de nuestras sociedades le ha permitido generar su propio proceso identitario que se concretiza en los constructos epistemológico, teórico y metodológico. 
- Respecto al situado histórico de la Psicología Social Comunitaria en Colombia encontramos que pese a ciertos esfuerzos que se han realizado en este sentido, todavía estamos en deuda de realizar una indagación profusa y profunda de su devenir.

- Contrario a los abundantes registros del ámbito de actuación de la Psicología Social Comunitaria en América Latina, en Colombia no se encuentra documentación suficiente sobre experiencias comunitarias significativas que hayan contribuido con el desarrollo de esta disciplina en los contextos locales y regionales.

- Lo anterior indica que es necesario adelantar investigaciones académicas de experiencias comunitarias significativas que le aporten a la construcción de la Psicología Social Comunitaria en nuestro país.

- Se necesita reconstruir las experiencias comunitarias significativas e identificar elementos de organización, participación e interacción en que haya colaborado la Psicología Social Comunitaria.

\section{REFERENCIAS BIBLIOGRÁFICAS}

\section{AGGLETON, P.,R. PARKER Y M. MALUWA. 2002. STIGMA, DISCRIMINATION AN} BUVINIÉ. (2004) in latin America and the Caribbean. Trabajo presentado en el seminario "HIV/AIDS" and Development. Reunión anual del Directorio Ejecutivo del Banco Interamericano de Desarrollo, 8 de marzo, Fortaleza, Brasil.

ARANGO, C. C. Historia de la psicología Comunitaria en Colombia En: http://www.ascofapsi. org.co/documentos/2007/hist_psico_comunitaria_col 2006.pdf. Consultado. Marzo 15 de 2008.

ARIAS, O. YAMADA Y L. TEJERINA. (2002). Education, Family Background and Racial Earnings Inequality in Brazil. Documento de trabajo de la Unidad de Pobreza y desigualdad, Departamento de Desarrollo sostenible del BID, Washington, D.C.

ASHMORE, M. (1989) The reflexive thesis: wrighting sociology of scientific knowledge. Chicago: Chicago University Press.

BANCO INTERAMERICANO DE DESARROLLO. Inclusión social y desarrollo en América Latina.Editado por Mayra Buvinié, Jacqueline Mazza y Juliana Pungiluppi con Ruthanne Deutsch.

BELLAMY, C.D. Y MOWBRAY, C.T. (1998) Supporting education as an empowerment intervention for people with mental illness. Journal of community psychology, 26 (5) 401- 414.

BERGER, P. Y LUCKMAN, T. (1967), La construcción social de la realidad, Madrid: Amorrortu-Murguía, 1986. 
BOJÓRQUEZ, N. Oficial del Programa de Derechos de la Niñez y la Mujer, UNICEF México.

BOUDON, R. (1993/1977) Effects pervers et ordre social. Paris: P.U.F.

BOURGUiGnON, F. (1999). Absolute Poverty, Relative Deprivation and social Exclusion. Trabajo presentado en el Taller del Informe de Desarrollo Mundial, Cpacity Building Internacional, 2-3 de febrero, Villa Borsig, Berlin.

BUSTELO, G, E. (1999). Pobreza moral. Reflexiones sobre la política social amoral y la utopía posible, en Bhattacharjea, S., Infancia y Política Social, UAM-UNICEF, México.

BUSTELO, G, E. (1998). Expansión de la ciudadanía y construcción democrática, en Todos entran. Propuesta para sociedades más incluyentes, UNICEF, Santillana. Colombia.

ESCOVAR, L.A. (1979) Análisis comparado de dos modelos de cambio social en la comunidad.

ESCOVAR, L.A. (1980) Hacia un modelo psicosocial del desarrollo. Boletín de AVEPSO, III (1) 1-6.

FERULLO DE P, A. (2006): El triángulo de las tres «p». Psicología, participación y poder, Buenos. Aires, Paidós.

FRANCO, A. (2003). Pobreza y desarrollo local. Colección de documentos del Instituto Internacional de Gobernabilidad. Proyecto LAGNIKS, www.iigov.org/biblioteca. IIG. España.

FREIRE, P. (2002): Educación y cambio, 5.a ed., Buenos Aires, Galerna-Búsqueda de Ayllu.

FREIRE, P. (1970). Pedagogía del oprimido. Montevideo: Tierra Nueva.

GARFINKEL, H. (1967) Studies in Etnomethodology. Englewood Cliffs, NJ: Prentice Hall.

GERGEN, K. (1973), La Psicología Social como historia. Anthropos, 177, 39-48.

GERGEN, K. (1991), El yo saturado. Dilemas de identidad en el mundo contemporáneo. Barcelona: Paidós, 1992.

GERGEN, K. (1994), Realidades y Relaciones. Aproximaciones a la construcción social. Barcelona: Paidós, 1995.

GERGEN, K. (1999) An invitation to social construction. London: Sage. 
GERGEN, K. (1982), Toward Transformation in Social Knowledge, London: Sage, 1994.

GADAMER, H.G. (1960), Verdad y método. Salamanca: Sígueme, 1991.

GERGEN, K. (1973), La Psicología Social como historia. Anthropos, 177, 39-48.

GERGEN, K. (1991), El yo saturado. Dilemas de identidad en el mundo contemporáneo. Barcelona: Paidós, 1992.

GERGEN, K. (1994), Realidades y Relaciones. Aproximaciones a la construcción social. Barcelona: Paidós, 1995.

GERGEN, K. (1999) An invitation to social construction. London: Sage.

GERGEN, K. (1982), Toward Transformation in Social Knowledge, London: Sage, 1994.

GRAY - MOLINA, G. PÉREZ DE RADA Y C. SOJO. (2003). Residential Segregation in Bolivia Cities. En J. R. Behrman, A. Gaviria y M. Székely (eds.), Who's In and Who's Aut: Social Exclution in Latin America. Washinton, D.C.: Banco Interamericano de Desarrollo.

GORE, C. (1995). Introduction: Markets, Citizenship and Social Exclusion. En G.

HERNÁNDEZ, E. (1994): «Elementos que facilitan o dificultan el surgimiento de un liderazgo comunitario», en M. Montero (coord.): Psicología social comunitaria. Teoría, método y experiencia, Guadalajara, Ediciones de la Universidad de Guadalajara.

IBÁÑEZ, T. (Comp.) (1989) El conocimiento de la realidad social. Barcelona: Sendai.

IBÁÑEZ T. (1990), Aproximaciones a la psicología social. Barcelona: Sendai.

IBÁÑEZ, T. (1994), Psicología social construccionista. Guadalajara (México): Universidad de Guadalajara.

IBÁÑEZ, T. (1996), Fluctuaciones conceptuales en torno a la postmodernidad y la psicología. Caracas: Universidad Central de Venezuela.

IBÁÑEZ, T . (1996), Fluctuaciones conceptuales en torno a la postmodernidad y la psicología. Caracas: Universidad Central de Venezuela.

IBÁÑEZ, T. (1997) Why a Critical Social Psychology? En T.Ibáñez y L.Íñiguez (Eds.) Critical Social Psychology. London: Sage. 27-41.

IBÁÑEZ, T. E ÍÑIGUEZ. (Eds.) (1997) Critical Social Psychology. London: Sage. 
KIEFFER, C. H. (1984) Citizen empowerment. Development perspective. Prevention In human services, 3, 9-36.

MARÍN, G. (1980). Hacia una Psicología Social Comunitaria. Revista Latinoamericana de Psicología, 12 (1), pp. 171-180. La psicología comunitaria en América Latina Revista «Poiésis». FUNLAM. N 15 - Junio de 2008. http://www.funlam.edu.co/poiesis

MARSHALL, T. H. (1950). Citizenship and Social Class. Ensayo.

MEAD, G.H. (1934). Espíritu, persona y sociedad. México: Paidós. 1993.

MONTERO, M. (2007). Aportes de la Psicología Comunitaria. Problemática de la actualidad latinoamericana. Compilación. JVG. Ediciones.

MONTERO, M. (Comp.). (1994). Psicología Social Comunitaria. México: Universidad de Guadalajara.

MONTERO, M. (Coordinadora). (1987). Psicología Política Latinoamericana. Venezuela: Editorial PANAPO.

MONTERO, M. (1994). “Un paradigma para la Psicología Social. Reflexiones desde el quehacer en América Latina”. En Construcción y crítica de la psicología social. Barcelona: Editorial Anthropos.

MONTERO, M. (2004). Introducción a la Psicología Comunitaria. Buenos Aires: Ed. Paidós.

MONTERO, M. (2000). Reflexiones sobre los fundamentos éticos de la Psicología Comunitaria. Universidad Central de Venezuela. En Modelos de Psicología Comunitaria para la promoción de la salud y prevención de enfermedades en las Américas. OPS. OMS. Salud y Sociedad. 2000. No7.

MONTERO, M. (1984). La Psicología Comunitaria: Orígenes, principios y fundamentos teóricos. Revista Latinoamericana de Psicología, Caracas, 16 (3), pp.387-400.

NEWBROUGH, J. R. (1992) "Community Psychology for the 1990"s" Journal of community psychology, 20, 1, 7-15.

ORAISÓN, M,. PÉREZ,A. M. (2006). Escuela y participación: El difícil camino de la construcción de ciudadanía. Artículo. Revista Iberoamericana de educación n. ${ }^{\circ}$ 47. pp. 15-29.

PELÁEZ R, M., CANON, O, NOREÑA, N, M. La intervención psicosocial en un contexto investigativo: "Lecturas psico-sociales sobre jóvenes agrópolis- sector rural - desde diversos actores que los intervienen”. Aletheia, jun. 2007, no.25, p.191-202. ISSN 1413-0394. 
POTTER, J Y WETHERELL, M. (1987), Discourse and Social Psychology. Beyond Attitudes and Behaviour, Londres: Sage.

POTTER, J. (1996), La representación de la realidad. Discurso, retórica y construcción social, Barcelona: Paidós, 1998.

RODGERS, C, GORE Y J. B. FIGUEIREDO. (eds.), Social Exclusion; Rhetoric Reality Responses. Ginebra, Internacional Institute for Labour Studies. Link, B. G. y Phelan (2002). On Stigma and its Public Health Implications. Trabajo presentado en la conferencia "Stigma and GlobalHealth: Developing a Research Agenda" (Estigma y salud global: desarrollo de una agenda de investigación), 5.7 se septiembre, Maryland.

RODGERS, G., C. CORE Y J. B. FIGUEIREDO (eds.) 1995. Social Exclusion: Rhetoric Reality Responses. Ginebra: Internacional Institute for Labour Studies.

SEN, A. (1999). Development as Freedom. Nueva York: Alfred A. Knopf, 2000, Social Exclusion: Concept, Application, and Security, Social Development papers 1. Banco Asiático de Desarrollo, Manila.

SHOTTER, J. (1987) The rhetoric of theory in psychology. Current issues in theoretical psychology. Pp.283-296.

SHOTTER, J. (1993a), Cultural Politics of Everyday Life, Buckingham: Open University Press.

SHOTTER, J. (1993b), Conversational Realities, Londres: Sage.

RODRÍGUEZ K, A. (1990). Estado Actual de la Investigación Psicosocial en "nuestra" América. Apuntes Psicología Social, Universidad Nacional de Córdoba. aforcada, E. (1992). Enfoque ecológico contextualista. Psicología Comunitaria. Centro Editor de América Latina, pp.26-34.

SERRANO GARCÍA, I. Y OTROS. (1992). Contribuciones Puertorriqueñas a la Psicología Social Comunitaria. EDUPR, San Juan de Puerto Rico.

TSAKLOGLOU, P Y F. PAPADOPULUS. (2001). Identifying Population Group at High Risk of Social Exclusion: Evidence from the ECHP. Documento para el debate 392. Institute for de study of labor.

VARGAS, V. (1997). Reflexiones en torno a los procesos de autonomía y la construcción de una ciudadanía femenina democrática en la región. P. 62, en Feminismo en transición y transición con feminismo. Memoria del Foro Internacional sobre Ciudadanía, Género y Reforma del Estado, GEM. 
VÁZQUEZ, F. (1998). Vivir con el tiempo en suspenso: notas de trabajo sobre transiciones políticas, memorias e historia. Anthropos, 177:67-72.

WIESENFELD, E Y SÁNCHEZ, E. (1991) "Introduction: the why, what and how of community social psychology in Latin America". Applied Psychology: An International Review. 40, 2.

WIESENFELD, E Y SÁNCHEZ, E. (En prensa) (Coords.), "Contribuciones latinoamericanas a la psicología social comunitaria" Caracas, Monte Avila Editores. 